\title{
LA DIMENSIÓN PRAGMÁTICA DE LA LINGÜÍSTICA DEL TEXTO
}

\author{
Belén SaIz Noeda \\ (Universidad de Alicante)
}

\begin{abstract}
RESUMEN
In this essay, I want to stress the relevance of Pragmatics to the evolution and consolidation of Text Linguistics from a comprehensive understanding of the text as a comunicative entity. The pragmatic dimension of Text Linguistics is essential and achieves its concreteness in the textual theories or models analyzed herein, all of which —especially those of Teun A. van Dijk and János S. Petofirepresent the most elaborate stage of Text Linguistics, namely due to the ultimate incorporation of the contextual (social and psycological) and communicative elements of language. Furthermore, it is through Pragmatics as an integration of both Syntax and Semantics that we reach the semiotic dimension of the text while at the same time we can propose a new conception of the linguistic science. And at the beginning and at the end of Pragmatics and Text Linguistics lies Rethoric, the ancient and eternal science of language.
\end{abstract}

[...] reconocemos que la lengua sin habla no tiene existencia real en ninguna parte; sólo existe en el uso activo que de ella hace el que habla o en el uso activo del que comprende. Sólo el «habla» real da realidad a la «lengua». Esto obliga a ver en el habla y no en la lengua el gozne de la ciencia del lenguaje.

(Amado Alonso, Prólogo a F. de Saussure, Curso de Lingüística General (1945), p. 30.)

\section{Sobre Lingüística del texto}

Tomando como principio y fundamento el presupuesto básico de que no hablamos por palabras o frases sino por textos ${ }^{1}$, la Lingüística del texto surgió y

1 El reconocimiento explícito del texto como unidad básica de la comunicación lingüistica, y de este hecho como principio fundamental de la linguística del texto lo encontramos en multitud de trabajos de los principales estudiosos de la lingüística textual. Por citar sólo tres ejemplos, cf. W. U. Dressler, Introduzione alla linguistica del testo, Roma, Officina, 1974, p. 12; A. García Berrio - A. Vera Luján, Fundamentos de teoría lingüística, Málaga, Comunicación, 1977, p. 171; T. Albaladejo, «On Text Linguistic Theory», en J. S. Petöfi (ed.), Text versus Sentence. Continued, Hamburgo, Buske, 1981, p. 1. 
se desarrolló ante la necesidad de dar cuenta de determinados fenómenos lingüísticos para los cuales las gramáticas oracionales no podían proporcionar explicaciones adecuadas. Desde su nacimiento, que se sitúa en Alemania en torno a los años sesenta ${ }^{2}$ - si bien es posible encontrar trabajos muy tempranos que, de manera dispersa y desde perspectivas diversas, ponen sus miras en el texto-, la lingüística textual se fue consolidando en torno al 'texto', unidad de delimitación compleja y extensión variable; el texto como dominio natural de una teoría del lenguaje ${ }^{3}$ y unidad lingüística básica ${ }^{4}$; como «conglomerado total de intención comunicativa $»^{5} \mathrm{y}$, sobre todo, la consolidación de la ligüística textual corrió pareja a la del texto como unidad comunicativa plena. Precisamente esta consideración plena del texto es fruto del encuentro entre Lingüística del texto y Pragmática, lejos de los inicios de la lingüística textual, todavía anclados en la oración, en los cuales el texto no era más que sucesión o concatenación de oraciones.

La Lingüística del texto, que procede directamente de la gramática generativotransformacional y del estructuralismo linguiístico, es sabido que reúne también otras aportaciones (tanto de disciplinas y escuelas en general como de contribuciones individuales, internas y externas a la Ciencia lingüística), y puede decirse que encuentra en la Retórica y la Poética clásicas sus más remotos y dos de sus principales antecedentes, y reconoce, por lo demás, su deuda con la Teoría y la Crítica literarias y la importancia de los frutos derivados de su estrecha colaboración con ella ${ }^{6}$.

2 Recordemos que la expresión «lingüística del texto» apareció por primera vez en 1955, en el artículo de Eugenio Coseriu titulado «Determinación y entorno» (E. Coseriu, «Determinación y cntorno. Dos problemas de una lingüística del hablar», en Teoria del lenguaje y lingüística general, Madrid, Gredos, 1973, p. 289, publicado por primera vez en Romanistisches Jahrbuch, VII, 195556 , pp. 29-54). Con el sentido que tiene actualmente, el primero en utilizar el término correspondiente en alemán, «Textlinguistik», fue Harald Weinrich en 1967 (H. Weinrich et alii, «Syntax als Dialektik (Bochumer Diskussion)», en Poetica, 1, 1967, pp. 109-126).

$3 \mathrm{Cf}$, por ejemplo, H. Hartmann, «Texte als linguistisches Objekt», en W. D. Stempel (ed), Beiträge zur Textlinguistik, Munich, Fink, 1971, pp. 10 y ss.

Vid. nota 1.

5 A. García Berrio-A. Vera Luján, Fundantentos de teoría lingiiística, cit., p. 172.

- La lingüística textual es para Tomás Albaladejo «la corriente teórico-lingüística que en mayor medida facilita y favorece la aplicación a la obra literaria de teorías lingüísticas elaboradas previamente con finalidad general de análisis lingüístico», sucediendo que «durante la realización y evolución de la teoría y crítica imnanentistas ha sido normal la asunción de la textualidad de la obra literaria, alcanzándose en este ámbito de la actividad filológica la unidad texto antes que en la lingüística teórica» (T. Albaladejo, «Sobre linguiística y texto literario», en Actas del III Congreso Nacional de Linguística Aplicada, Valencia, 16-20 de abril de 1985, Univ. de Valencia, 1986, pp. 39-40). De las relaciones entre la Lingüistica del texto y la teoria literaria materializadas en la Poética lingüistica de base textual se ocupa con detalle F. Chico Rico, «Lingüística del texto y Teoria literaria», en Rilce. Revista de Filología Hispánica, 8, 2, 1992, pp. 226-264; yo misma me he ocupado del papel de los estudios literarios como antecedente, a la vez que como factor fundamental del desarrollo de la Lingüística textual: B. Saiz Noeda, De la gramática transfrástica a la lingǘstica comunicativa: nacimiento y desarrollo de la Lingüistica del texto, Alicante, Universidad, 1993 (microfichas), pp. 38-58. 
Las primeras tentativas transfrásticas, llevadas a cabo tanto en el ámbito europeo como en el americano desde presupuestos básicamente estructuralistas ${ }^{7}$, más que definir el texto en sí, se dedicaron a analizar de qué modo se conectan unos enunciados con otros, manteniendo la oración, por consiguiente, su papel de unidad lingüística básica. El instrumental teórico generativista sirvió, pues, de marco y medio para el desarrollo de los primeros intentos de gramáticas textuales y de definición de la unidad textual en tanto que unidad semántica global. Sin duda, a lo largo de la historia de la lingüística textual ha sido cada vez mayor la importancia concedida a los factores contextuales, a medida que se iba haciendo más patente la imposibilidad de describir adecuadamente el 'texto', sin tomar en consideración los aspectos contextuales o pragmáticos. De ahí que la inclusión del contexto en la Lingüística (o la consideración del texto, unidad lingüística básica, en su contexto) fuera un paso decisivo para que la lingüística textual evolucionase. En palabras de Graziella Tonfoni, «el processo dell'espansione teorica testuale che procede [...] da una fase sintattica, e attraverso una espansione semantica, giunge all' ambito pragmatico» ${ }^{8}$.

\section{2. ... y Pragmática}

Con el término pragmática se presenta una disciplina o campo de estudio cuyo objeto y límites no están claramente definidos, dada la diversidad de orientaciones, corrientes de investigación o disciplinas, que se reúne bajo este

Dentro del estructuralismo americano, es preciso destacar la aportación de Zellig S. Harris, cuyo «Análisis del discurso» puede ser considerado el primer impulso a la moderna Lingüística del texto (Z. S. Harris, «Discourse Analysis», en Language, 28, 1952, pp. 1-30. En el ámbito europeo, además de Eugenio Coseriu con su ya citado «Determinación y entorno», son especialmente dignos de mención los trabajos realizados en Alemania por Peter Hartmann: «Text, Texte, Klassen von Texten», en Bogawus. Zeitschrift für Literatur, Kunst und Philosophie, 2, 1964, pp. 15-25; «Texte als linguistisches Objekt», cit., pp. 9-29; «Textlinguistik als linguistische Aufgabe», en W. U. Dressler (ed.), Textlinguistik, cit., pp. 93-100 (publicado por primera vez en S. J. Schmidt (ed.), Konkrete Dichtung. Konkrete Kunst, Karlsruhe, Privatdruck, 1968, pp. 62-77); «Zur Aufgabe der Textlinguistik», en Lingua, 21, 1968, pp. 197-2 15; «Zum Begriff des sprachlichen Zeichens», Zeitschrift für Phonetic, Sprachwissenschaft und Kommunikationsforschung, 21, 3/4, 1968, pp. 205-222; «Textlinguistik als neue linguistische Teildisziplin», en Replik, 1, 2, 1968, pp. 2-7; «Probleme der semantischen Textanalyse», en S. J. Schmidt (ed.), Text, Bedeutung, Asthetik, Munich, Bayerische Schulbuch, 1970, pp. 15-42; «Texte als linguistisches Objekt», cit.; «Textlinguistische Tendenzen in der Sprachwissenschaft», en Folia Linguistica, VIII, 1, 4, 1975, pp. 1-49). Peter Hartmann será después el principal animador del Círculo de Constanza, que reunirá a estudiosos como J. F. Ihwe, H. Rieser y I. S. Petöfi en el primer proyecto de una gramática textual (vid., a este respecto, J. Ihwe - H. Rieser - W. Köch - M. Rüttenauer, «Informationen über das Konstanzer Projekt 'Textlinguistik'», en Linguistiche Berichte, 13, 1971, pp. 105-106; T. A. van Dijk - J. Ihwe - J. S. Petöfi - H. Rieser, Zur Bestimmung narrativer Strukturen auf der Grundlage von Textgrammatiken (Papiere zur Textlinguistik 1), Hamburgo, Buske, 1972). Puede verse una reconstrucción histórica de la Lingüística del texto en mi estudio De la gramática transfrástica a la lingüistica comunicativa: nacimiento y desarrollo de la Lingǘstica del texto, cit.

8 G. Tonfoni, Dalla linguistica del testo alla teoria testuale, Milano, Unicopli, 1983, p. 25. 
rótulo, lo cual ha tenido como consecuencia una considerable multiplicidad en los intentos de definición de la Pragmática -de acuerdo con las múltiples perspectivas que integra- ${ }^{9}$. El sentido etimológico de la palabra, del griego $\pi \rho \alpha \gamma \mu \alpha, \pi \rho \alpha \gamma \mu \alpha \tau o s$, 'acción, ejecución' ( $\pi \rho \alpha \sigma \sigma o$, 'actuar, realizar'), queda recogido en la Pragmática como estudio del lenguaje en tanto que acción: qué se hace al decir algo, o cómo hacer cosas con palabras, según reza el título del conocido trabajo de John L. Austin ${ }^{10}$, cuya teoría de los actos lingüísticos, junto a la «teoría de los actos de habla» de su continuador, el americano John Searle ${ }^{11}$, pone de manifiesto que el lenguaje es también una forma de conducta $y$, de este modo, se cuenta entre los trabajos no lingüísticos fundacionales de la Pragmática -aún sin nombrarla-. Para Searle «hablar una lengua es tomar parte en una forma de conducta (altamente compleja) gobernada por reglas»; el uso del lenguaje, como el juego, está regido por reglas, las cuales es preciso dominar para dominar una lengua ${ }^{12}$. Aun cuando parece poco probable que influyeran en el desarrollo de las ideas de Austin las Investigaciones filosóficas de Wittgenstein ${ }^{13}$, es innegable el paralelismo existente entre la teoría de los actos de habla de Austin y Searle y la «teoría del juego del lenguaje» del filósofo austriaco. Su aportación, fundamental, a la Pragmática, como las de Austin y Searle, se inscribe en el ámbito de la filosofía del lenguaje. Según Wittgenstein, el significado de una palabra es su uso -individual, libre, a la vez que socialmente regulado-en el lenguaje; las expresiones verbales adquieren su sentido únicamente en relación con las actividades, o «juegos lingüísticos», de las que forman parte.

En la línea del «significado en el uso» de Wittgenstein se encuentra una de las más sugerentes concepciones de cuantas se han ofrecido sobre la Pragmática, la de Peirce. Para Peirce la Pragmática o «retórica pura», que se asocia a la Sintaxis y la Semántica como tercera rama de la Semiótica o ciencia general de los signos, tiene por cometido «determinar las leyes mediante las cuales [...] un signo da nacimiento a otro signo $\mathrm{y}$, especialmente, un pensamiento da naci-

9 Queda fuera de los límites del presente trabajo abordar todas y cada una de las posibles definiciones de la pragmática. Con relación a estas cuestiones resulta imprescindible recordar a $S$. C. Levinson, quien en 1983, en el que se ha considerado el «libro de la disciplina», su Pragnatics, Cambridge, Cambridge University Press, of rece catorce distintas definiciones de la pragmática, lista a la que hoy sin duda se podrían añadir muchas más. También de 1983 e igualmente de necesaria mención en este punto es el trabajo de G. Leech, Principles of Pragmatics, Londres/New York, Longman. Vid. además: M. Bertucceli, (1993), Qué es la Pragmática. Barcelona, Paidós, 1996; M. Victoria Escandell, Introducción a la Pragmática, Barcelona, Anthropos, 1993, pp. 1547 .

10 J. S. Austin, (1962), Cómo hacer cosas con palabras. Palabras y acciones, Barcelona, Paidós, 1982.

\footnotetext{
"I.Searle, (1969), Actos de habla. Ensayo de filosofia del lenguaje, Madrid, Cátedra, 1980.

12 J. Searle, ibid., p. 22.

13 L. Wittgenstein, (1953), Investigaciones filosóficas, Madrid, Crítica, 1988.
} 
miento a otro pensamiento» ${ }^{14}$. La difusión de la tríada semiótica peirceana y, en particular, de la Pragmática fue labor de Charles Morris ${ }^{15}$, para quien este tereer componente de la Semiótica, que engloba los otros dos, se ocupa de las relaciones existentes entre los signos y sus usuarios.

Sea cual fuere la perspectiva adoptada por cada una de las orientaciones que la Pragmática engloba, el denominador común a todas ellas es su preocupación por las relaciones entre las expresiones lingüísticas, los participantes comunicativos y el contexto en el que aparecen, o, lo que es lo mismo, la consideración de «el lenguaje en usos ${ }^{16}$.

Entre las contribuciones esenciales a las investigaciones pragmáticas, quiero referirme brevemente a la Retórica clásica, ya mencionada anteriormente como antecedente remoto y fundamental de la lingüística textual. En tanto que arte de la persuasión por la palabra, la Retórica tiene como finalidad la consecución de un discurso, con apariencia de verdad, que sea eficaz: capaz de emocionar, conmover (movere) al juez y/o al auditorio en favor de la causa defendida. Para la Retórica, pues, hablar, esto es, pronunciar un discurso lleva implícito el sentido de 'acción', de 'ejecución' al que etimológicamente remite la palabra "pragmática»; sentido que se concreta y se hace manifiesto a través de la actio o pronuntiatio, la operación retórica pragmática por excelencia, la de la «ejecución» del discurso, que toma en consideración no sólo la voz y la entonación, sino también los gestos, los movimientos y hasta la indumentaria del orador: en la actio se funden el «actuar» en el discurso (efectivamente realizado) y por el discurso, como materialización de la intención del orador de captación de la voluntad del juez y/o el público oyente. Por otro lado, la gran Retórica incluye el estudio del carácter y de las pasiones humanas como fuentes de la persuasión (psicagogía). En tanto que ciencia del lenguaje, del discurso, más que ninguna otra disciplina es la Retórica ciencia del «lenguaje en uso». Y en general, la Retórica considera el discurso en sí, la res y los verba que lo constituyen, siempre en relación con los participantes comunicativos, el contexto en el que se inserta y los demás elementos de la comunicación, de modo que

${ }^{14}$ Ch. Peirce (ed.), La ciencia de la semiótica, Buenos Aires, Nueva Visión, 1974, p. 23.

is Ch. Morris, (1938), «Fundamentos de la teoría de los signos», en F. Gracia (comp.), Presentación del lenguaje, Madrid, Taurus, 1972, pp. 53-65.

16 Para la pragmática en general, $y$, concretamente, sobre las distintas aportaciones, desde ámbitos y perspectivas muy diversos, que confluyen en la pragmática lingüística y para una reconstrucción histórica de la misma, vid. E. Bernárdez, Introducción a la Lingüística del texto, Madrid, Espasa Calpe, 1982, pp. 53-74; G. Leech, Principles of Pragmatics, cit.; S. Levinson, Pragmatics, cit.; M. E. Conte, «La pragmatica linguistica», en C. Segre, Intorno alla linguistica, Milano, Feltrinelli, 1983, pp. 94-96; A. Herrero, Signo/Texto, Alicante, Universidad, 1986, pp. 69-141; F. Chico, Pragmática y construcción literaria, Alicante, Universidad, 1987, pp. 31-63; J. M. Jiménez Cano, «Problemática metodológica en el análisis de los fenómenos textuales pragmáticos», en Anales de la Universidad de Murcia, 1-2, XL1, I983, pp. 299-370; B. Schlieben-Lange, (1974), Pragmática lingiuística, Madrid, Gredos, 1986; G. Reyes, La pragmática lingüística, Barcelona, Montesinos, 1990; M. Bertuccelli, Qué es la pragmática, cit.; M. V. Escandell Vidal, Introducción a la pragnática, cit. 
su identificación con la Pragmática puede ser casi absoluta ${ }^{17}$.

La consideración del lenguaje en el contexto implica el acercamiento a lo social del lenguaje. Al dar cuenta la Pragmática de la dimensión social y comunicativa del lenguaje, de la misión de éste de contribuir a la comunicación y a la interacción social ${ }^{18}$, entra en relación con la Sociología y se encuentra estrechamente vinculada a la Sociolingüística o «lingüística de orientación social $\gg^{19}$ la cual (como sucede — de otro modo, desde otra perspectiva-con la lingüística textual) representa la respuesta a una doble necesidad sentida en el propio seno de la Lingüística: de ampliación de los horizontes, como superación del exceso formalista, y de mayor realismo social. En tanto que disciplina atenta a la interacción entre el uso del lenguaje y la organización social del comportamiento, se ocupa de cómo se habla una lengua, cómo se desarrolla la práctica comunicativa y de qué parámetros depende. A medida que la Pragmática se hace más empírica, cabe decir, se aleja de sus orígenes filosófico-formales y se acerca a la Sociolingüística ${ }^{20}$.

De entre los «usos del lenguaje» en los que la Pragmática se ha adentrado y con cuyo estudio ha progresado destaca el literario. Al igual que sucediera en el ámbito de la Lingüística, la Pragmática vino a ampliar los horizontes formalistas de la Ciencia literaria, una vez que el acercamiento restrictivamente inmanentista a la literatura, asumida ya la textualidad de la obra literaria, había tocado techo sin llegar a proporcionar explicaciones satisfactorias del fenómeno literario. La Pragmática literaria supone, por un lado, el tratamiento de la obra literaria, del texto literario como un acto de habla de características especiales, el acto de comunicación literaria; ello implica la consideración del texto en su contexto, con todo lo que ello conlleva ${ }^{21}$. Por otro lado, desde un

17 Cf. B. Moratara Garavelli (1988), Manual de Retórica, Madrid, Cátedra, 1991, que señala, por ejemplo, la deuda que una teoría pragmática como la de las «máximas conversacionales» de $\mathrm{H}$. Paul Grice tiene para con la teoría retórica de las virtudes del discurso (pp. 77-79). Para los conceptos básicos de la Retórica, vid. H. Lausberg, (1960), Manual de Retórica literaria, Madrid, Gredos, 1966-1968, 3 vols. Vid. también A. Reyes, (1941), La crítica en la edad ateniense. La antigua retórica (Obras Completas, XIII), México, Fondo de Cultura Económica, 1983 (reimpr. de $\left.1961^{i}\right)$.

${ }_{18}$ T. A. van Dijk, (1978), La ciencia del texto: un enfoque interdisciplinario, Barcelona, Paidós, 1983, p. 79.

19 J. A. Villena Ponsoda, Fundamentos del pensamiento social sobre el lenguaje (Constitución y Crítica de la Sociolingüistica), Málaga, Ágora, 1992, p. 29.

20 Sobre la Sociolingüística, su constitución, sus límites, vid. ibid. pp. 15-79. Para las relaciones entre Pragmática y Sociolinguística, vid. G. Reyes, La pragmática lingüística, cit., pp.34-38.

21 «La pragmática de la comunicación literaria deberá tratar de los siguientes tipos de temas y problemas: i) ¿Qué tipo o tipos de acción se llevan a cabo en la producción de textos literarios? ii) ¿Cuáles son las condiciones de propiedad de dichas acciones? iii) ¿Cuál es la estructura del contexto en cuyos términos se define la propiedad? iv) ¿Cómo se relacionan las «acciones literarias» y su contexto con las estructuras del texto literario? v) ¿En qué medida estas acciones, contextos y manifestaciones textuales son semejantes y/o diferentes a los de otros tipos de comunicación, tanto 
punto de vista teórico-literario, el estudio desde presupuestos pragmáticos de la literatura apunta como criterio a la hora de definir la literariedad de la obra de arte verbal una convención de uso de naturaleza social» ${ }^{22}$. Según esto, la literatura es aquello que una comunidad de lectores determina como tal de acuerdo con factores de tipo histórico, sociológico, lingüístico, etc.; o bien los lectores deciden que un texto es literario por motivos que residen en el propio texto y que tienen que ver con la utilización sistemática de recursos que conducen al «extrañamiento», lingüística y comunicativamente hablando. Desde la llamada Poética linguística textual de base pragmática, hasta la crítica psicoanalítica, la crítica sociológica, la Teoría de la recepción, la Ciencia empírica de la literatura, la Poética de lo imaginario..., muchas son las orientaciones y modelos teóricos y/o críticos que, focalizando uno o varios (todos en el caso de la Pótica lingüística) componentes del esquema comunicativo, se acercan a la literatura desde un punto de vista pragmático. Sin embargo, tampoco la exclusiva consideración del texto literario como acto comunicativo proporciona respuestas satisfactorias a cuestiones que atañen a la esencia literaria. No en vano nos hallamos ante la más compleja y menos «pragmática» de las realizaciones lingüísticas ${ }^{23}$.

\section{Sobre Pragmática y Lingüística del texto}

Mientras la Pragmática da cuenta de la dimensión social y comunicativa del lenguaje, la Linguística del texto, por su parte, puesto que se ocupa del lenguaje, el «lenguaje en textos», ha de atender también al lenguaje en tanto que comunicación; esto es, al texto como unidad comunicativa. Con la incorporación de la Pragmática a la Lingüística, ésta amplía sus horizontes: lo «lingüístico» y lo «extralingüístico» (o pragmático) se unen en el nivel textual, adquiriendo dimensión semiótica ${ }^{24}$. La 'competencia textual' es, de este modo, «competen-

verbal como no verbal? vi) ¿Qué problemas existentes tanto en la poética como en el funcionamiento real de la literatura en la sociedad pueden ser (re)formulados en términos de una teoría pragmática? [...] vii) ¿Cuál es la base cognitiva (emotiva, etc.) de las nociones pragmáticas mencionadas anteriormente (acción, propiedad, etc.)? viii) ¿Cuál es la base social y cultural de las nociones pragmáticas mencionadas anteriormente: qué convenciones, normas, valores, y qué estructuras de las sociedad vinculan la propiedad de la acción «literaria» con los procesos reales de aceptación, rechazo, etc.. de los textos literarios?» (T. A. van Dijk, «La pragmática de la comunicación literaria», en J. A. Mayoral (comp.), Pragmática de la contunicación literaria, Madrid, Arco, 1987, pp. 178-179).

22 J. M. Pozuelo Yvancos, (1988), Teoría del lenguaje literario, Madrid, Cátedra, 19944, p. 76.

23 Para las cuestiones de Pragmática literaria, vid. especialmente J. A. Mayoral (comp.), Pragnática de la comunicación literaria, cit.; vid. asimismo F. Chico Rico, Pragmática y construc:ción literaria, cit.

24 Recordemos que Charles Morris, gran difusor de la Pragmática, considera ésta como el tercer componente, globalizador de los otros dos, de la Semiótica o ciencia general de los signos que se ocupa de las relaciones existentes entre los signos y sus usuarios (Ch. Morris, «Fundamentos de la teoría de los signos», cit.). De la dimensión pragmática y semiótica del signo texto a la luz de la 
cia comunicativa»: «la capacità del parlante d'impicgare adeguatamente il linguaggio nelle varie situazioni ${ }^{25}$ ), y, por otro lado, la Pragmática lingüística se convierte en Pragmática lingüístico-textual, al llevarse a cabo la comunicación lingüística por medio de textos. «La pragmática se ocupa, pues, -en palabras de T. A. van Dijk - de la relación entre la estructura textual y los elementos de la situación comunicativa sistemáticamente ligados a ella: todos estos elementos juntos forman el contexto» ${ }^{26}$.

\subsection{Tipos de textos}

La profundización en el estudio de la Pragmática del texto viene determinada por la necesidad de resolver uno de los problemas básicos que se le plantean a la lingüística textual: la clasificación tipológica del texto. Cuando se intenta llevar los modelos abstractos de la lingüística textual a los diversos tipos de textos que constituyen el objeto de estudio de la Teoría y Crítica de la literatura, el Derecho, la Teología, etc., las gramáticas textuales «semántico-sintácticas» demuestran ser insuficientes ${ }^{27}$. Es a partir de la incorporación de la Pragmática a la Linguística el momento en que la lingüística textual puede establecer vínculos con otras disciplinas que trabajan con textos y aplicarse a otros campos $^{28}$. Se trata de buscar criterios para clasificar los textos comunes a la lingüística y a las demás «ciencias textuales». Elisabeth Gülich y Wolfgang Raible recogen, en Textsorten ${ }^{29}$, las distintas propuestas presentadas en un coloquio desarrollado en Bielefeld en 1972 en torno a este tema. Todas ellas apuntan la imposibilidad de fundamentar una tipología textual sobre criterios

doctrina peirceana -entre otras- se ocupa Ángel Herrero en Signo/Texto (De Gramática a Retórica), Alicante, Universidad, 1986. «El 'texto' - afirma Herrero-resulta signo por excelencia del intercambio semiótico, puesto que significa, o 'transporta', dicho intercambio, como fin, como satisfacción de su ocurrencia: objeto, por consiguiente, de toda posible retórica, nueva o vieja, que se establece, más allá de un marco semiótico determinado, como estudio de las condiciones de comunicación del significado, o 'retórica pura'» (Ibid., pp. 65-67). Con relación al análisis del texto como objeto de la Semiótica, vid. también J. Lozano - C. Peña-Marín - G. Abril, Análisis del discurso. Hacia una semiótica de la interacción textual, Madrid, Cátedra, 1982. Un concepto amplio de texto con un sentido plenamente semiótico es el que corresponde a la visión de la «Semiótica de la cultura» de J. M. Lotman y la Escuela de Tartu (vid. J. M. Lotman y Escuela de Tartu, Seniótica de la Cultura, Madrid, Cátedra, 1979).

25 M. E. Conte, «Introduzione», en M. E. Conte (ed.), La linguistica testuale, Milano, Feltrinelli, 1977, p. 21. En el mismo sentido afirma Ángel Herrero que «sólo integrando las tres dimensiones semióticas podrá hablarse de una auténtica competencia textual, como capacidad de identificación y empleo de textos [...]» (A. Herrero, Signo/Texto, cit. pp. 97 y 99).

26 T. A. van Dijk, La ciencia del texto, cit., p. 81.

27 M. Verlato, Avviamento alla linguistica del testo, Padova, CLESP, 1983, pp. 132 y ss..

28 «Only then [cuando se ha completado la gramática textual con un componente pragmático] does it provide a sound basis for psycho- and sociolinguistic theories of verbal performance and interaction, and only then can an adequate theory of literature be based or modelled on it», afirmaba ya van Dijk en sus Some Aspects of Text Grammar, The Hague, Mouton, 1972, p. 314.

20 E. Gülich - W. Raible (eds.), Textsorten. Differenzierungskriterien aus linguistischer Sicht, 
exclusivamente «internos al texto» (textintern) y la necesidad de recurrir a factores pragmáticos (textextern $)^{30}$. De entre las propuestas presentadas en este coloquio destaca la de Werner Kummer, quien, influido por la teoría de los actos de habla de Austin y Searle, reclama una «teoría general de la acción» y propone considerar el texto en tanto que secuencia de actos lingüísticos ${ }^{3 !}$.

\subsection{La teoría del texto de Siegfried J. Schmidt}

Frente a autores, como Wolfgang U. Dressler ${ }^{32}$, que consideran la Pragmática como un mero componente añadido a la gramática textual propiamente dicha cuya función consiste en insertar el texto en una situación comunicativa, Siegfried J. Schmidt representa a un grupo de estudiosos para los que la Pragmática se integra totalmente y como componente fundamental en la Teoría del texto, la cual se convierte de este modo en una Teoría pragmática del texto. En 1973 publica Schmidt su Texttheorie ${ }^{33}$, en la que reivindica el papel de la Pragmática y propone una reformulación de los estudios del texto sobre esa nueva base:

[...] la «pragmática» ha de liberarse hoy de su condición de «pariente pobre de la linguística» $\mathrm{y}$ ha de integrarse en el conjunto de la teoría lingüística.

[...] este proceso [...] supone un paso más en el camino de la lingüística, orientada hacia la realidad del lenguaje en la comunicación socio-verbal como sector central de investigación, con otras palabras, un paso de la lingüística del sistema verbal) hacia una teoría del texto como teoría de la comunicación verbal ${ }^{34}$.

De acuerdo con la teoría del «acto verbal»o de la «actividad verbal»cuyos fundamentos se encuentran en Austin y Searle y, muy especialmente en el caso de Schmidt, en los psicólogos y psicolingüistas soviéticos A. A. Leont'ev y A. N. Leont'ev ${ }^{35}$-, Schmidt considera que el lenguaje es un sistema de

Frankfurt, Athenaum Verlag, 1972. E. Bernárdez se ocupa de la tipología textual en su Introducción a la lingüística del Texto, cit., pp. 213-230.

30 E. Gülich - W. Raible, «Textsorten als linguistische Problem. Vorwort und Einleitung», en E. Gülich - W. Raible (eds.), Textsorten, cit., p. 3.

3 W. Kummer, «Aspects of a Theory of Argumentation», en E. Gülich - W. Raible (eds.), Textsorten, cit., pp. 25-49.

32 W. U. Dressler, Introduzione alla linguistica del testo, cit., pp. 131-142.

33 S. J. Schmidt, Teoría del texto. Problenas de una lingüística de la comunicación verbal, cit.; su «Teoria del testo e pragmalinguistica», en M. E. Conte (ed.), La linguistica testuale, cit., pp. 248-271, recoge los principales aspectos de dicho libro.

34 S. J. Schmidt, Teoría del texto, cit, p. 20.

35 A. A. Leont'ev, Sprache - Sprechen - Sprechtätigkeit, (versión alemana: Stuttgart, W. Kohlhammer, 1971). Para un mejor entendimiento de la Ilamada «teoría del acto verbal», vid. E. 
actividad (hablar como hacer lingüístico) que se integra con otros factores en el campo de una actividad comunicativa compleja - que a su vez forma parte de una actividad social de complejidad mayor- ${ }^{36}$, según aparece representado en el siguiente esquema en el que $\uparrow$ indica inclusión $)^{37}$ :

\section{Interacción social \\ $\uparrow$ \\ Comunicación verbal \\ $\uparrow$}

Actividad comunicativa en tipos de situaciones comunicativas $\uparrow$

Actos comunicativos

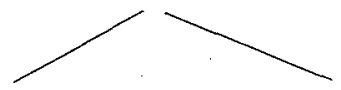

Constituyentes verbales Constituyentes no verbales

Schmidt entiende la 'textualidad' como «el modo universal y social de llevar a cabo la comunicación lingüística», siendo los 'textos' las realizaciones concretas de la 'textualidad', es decir, «porciones enunciadas de signos del lenguaje que funcionan comunicativamente», y, por tanto, «textos-en-función» ${ }^{38}$. La Teoría del texto, según Schmidt, tiene que analizar, pues, el lenguaje en el contexto socio-comunicativo y el lenguaje en textos. Schmidt sintetiza su propuesta de teoría textual en los siguientes términos:

El tema de investigación de una (lingüística como) teoría del texto consistiría, pues, en desarrollar una teoría explícita de la comunicación verbal. En tal teoría del texto [...] pragmático ya no puede ser aquí la calificación de un ámbito de investigación parcial, teóricamente aislable, sino a lo sumo, la calificación del aspecto investigador dominante de la teoría del texto en su conjunto [...]. El objeto de investigación de una teoría del texto sería, entonces, examinar más de cerca con qué medios y según qué reglas se producen y reciben textos en función ${ }^{39}$.

Así pues, la unidad básica de su teoría es el «juego de acción comunicativo» («das kommunikative Handlungsspiel», o «historia» comunicativa compleja),

Bernárdez, Introducción a la Lingüística del Texto, cit., pp. 59 y ss.

36 Recordemos el sentido etimológico de 'acción; actuar, ejecutar' de la palabra «pragmática», anteriormente expuesto.

37 S. Schmidt, Teoría del texto, cit., p. 56.

38 Ibid., p. 148.

39) Ibid., p. 25. 
que se analiza en términos de actos de comunicación o actos lingüísticos (Sprechakte), los cuales se realizan en forma de texto ${ }^{40}$. Como dice M. E. Conte, la teoría de Schmidt, más que una teoría del texto en el contexto, es una teoría del contexto del texto ${ }^{4 !}$.

\subsection{El modelo textual sistemático-funcional de M.A.K. Halliday}

La llamada gramática sistémico-funcional ha sido elaborada fundamentalmente por Michael A. K. Halliday a partir de las ideas de Firth, desde una perspectiva sociolingüística, asumiendo el punto de vista del «hombre social». Ruqaiya Hasan ha contribuido en gran medida al desarrollo del componente textual de dicha gramática. ${ }^{42}$

El componente textual del sistema construido por Halliday es el que permite la creación de textos, marcando así la diferencia entre lenguaje en sentido abstracto y lenguaje en uso; «[...] it is through the semantic options of the textual component that language comes to be relevant to its environment» ${ }^{43}$. Dicho componente textual corresponde a la «perspectiva funcional de la frase» de la Escuela de Praga ${ }^{44}$.

Las nociones de 'textura' y 'estructura' determinan el 'texto'. La textura es lo que define a una cadena de oraciones (o de unidades léxico-gramaticales) como texto, frente a las que no lo constituyen. La textura viene dada por la cohesión en el plano del significado $0^{45}$, la cual, a su vez, es el resultado de dispositivos lingüísticos tales son la referencia, la sustitución, la elipsis, etc., que dan lugar a los distintos tipos de cohesión. La estructura es lo que permite distinguir entre texto completo e incompleto, así como lo que determina la «fórmula estructural» o «estructura genérica», es decir, «the form that a text has

40 S. Schmidt, «Teoria del testo e pragmalinguistica», cit.; S. J. Schmidt, «Das 'Kommunicative Handlungsspiel' als Kategorie der Wirklichkeitskonstitution», en S. J. Schmidt (Hrsg.), Pragmatik I. Interdisziplinäre Beitrüge zur Erforschung der sprachlichen Kommunikation, München, W. Fink, 1974, pp. 103-117.

41 M. E. Conte, «Introduzione», cit., p. 49.

42 Cf. M. A. K. Halliday, «Estructura y función del lenguaje», en J. Lyons, Nuevos horizontes de la linguiistica, Madrid, Alianza, 1975; «Text as Semantic Choice in Social Contexts», en T. A. van Dijk - J. S. Petöfi (eds.), Granmars and Descriptions, Berlin/New York, Walter de Gruyter, 1977; M. A. K. Halliday - R. Hasan, Cohesion in English, London, Longman, 1976; R. Hasan, «Text in the Systemic-Functional model» en W. U. Dressler (ed.), Current Trends in Textlinguistics, Berlin/New York, Walter de Gruyter, 1978, pp. 228-246. Para una síntesis del citado modelo, vid: E. Bernárdez, Introducción a la Lingüística del Texto, cit., pp. 194-203; M. Verlato, Avviamento alla linguistica del testo, cit., pp. 154-165.

43 M. A. K. Halliday, «Text as Semantic Choice in Social Contexts», cit., p. 181.

44 Su concepción sobre la estructura tema-rema puede verse en ibid., pp. 181 y ss. 
as a property of its genre» ${ }^{46}$, lo cual tiene que ver con el tipo de texto y está fuera del sistema lingüístico: «it is language as the projection of a higher-level semiotic structure ${ }^{47}$ (vendría a coincidir con el concepto de 'superestructura' de van Dijk, del que brevemente trataré más adelante).

Las dos características básicas del 'texto' en el modelo sistémico-funcional son las siguientes:

-Por una parte, el texto es considerado como unidad semántica que se realiza superficialmente en oraciones.

-Por otra parte, está englobado en un marco semiótico-social y el texto es producto de su entorno.

De la conjunción de las dos se sigue que el texto es medio de intercambio entre los miembros de un sistema social. El significado se codifica por medio del lenguaje en forma de texto (en la medida en que representa una de las formas de realización de la semiótica social, la (socio-)semántica funciona como nivel intermedio entre el lingüístico y el no lingüístico). Más aún, el texto es un acontecimiento social a través del cual los significados que constituyen el sistema social se intercambian ${ }^{48}$.

En la medida en que el sistema semántico representa la realización de un potencial de comportamiento, el texto se define con una acción, «lengua en uso». Se trata además de un proceso continuo de relación entre un texto y su entorno; un proceso continuo de elección semántica. La «función textual» es aquélla que hace posible la «puesta en funcionamiento» de la lengua, estableciendo correspondencias en el interior de la misma y entre ella y el contexto, que tiene como resultado los textos (sería la equivalente a la «competencia comunicativa»).

Puesto que el texto es un fenómeno social, una forma de conducta social, el concepto de «situación» adquiere una dimensión fundamental. El contexto de la situación, que es una estructura semiótica (un «constructo social») no incluye al texto sino que lo determina, forma parte del texto dando cuenta de las «propiedades ecológicas» del lenguaje, es decir, los rasgos que lo relacionan con su entorno en el sistema social.

La relación entre el tex to y la oración en el modelo de Halliday rompe con la concepción de los «niveles» lingüísticos imperante. El texto no consta de oraciones, sino que se realiza o es codificado mediante oraciones, con lo cual

45 Cf. M. A. K. Halliday - R. Hasan, Cohesion in English, cit. La cohesión para ellos es uno de los criterios que diferencia un texto que funciona como un «todo integrado» de un conjunto de oraciones sin relación alguna.

46 M. A. K. Halliday, «Text as Semantic Choice in Social Contexts», cit., p. 192.

47 Ibid., p. 193.

48 «A text is a social event whose primary mode of unfolding is linguistic. If text can be seen as a bridge between the verbal symbolic system and the culture, this is because of the relationship between text and social context: text is 'in language' as well as 'in culture"» (R. Hasan, "Text in the Systemic-Functional Model», cit., p. 229). 
deja de tener sentido la oposición entre lingüística oracional y lingüística del texto. La cuestión esencial que se plantea en el modelo de Halliday ya no es la relación entre estructura profunda textual, «macroestructura» o «base textual», según se trate de la teoría de van Dijk o de la de Petöfi respectivamente - que veremos a continuación-, y estructura de superficie, sino la relación del texto, por una parte, con las oraciones que lo realizan y, por otra, con el nivel más alto de significado del que es realización; o, lo que es lo mismo, poner en relación texto y contexto.

\subsection{Las teorías textuales de Teun A. Van Dijk y János S. Petöfi}

Tanto Teun A. van Dijk como János S. Petöfi se han ocupado de la Pragmática en el marco de sus respectivas teorías textuales. Numerosos son los trabajos de T. A. van Dijk que se refieren a la Pragmática del discurso, y su propuesta teórica incluye explícitamente «macroestructuras pragmáticas» y «macroactos de habla». Por su parte, J. S. Petöfi diferencia, ya desde los primeros desarrollos de su «teoría de la estructura del texto y de la estructura del mundo» (TeSWeST) - según apunta la misma denominación de su teoría-, un componente «cotextual», o componente de gramática del texto, y un componente «contextual», o componente de semántica de mundo. Sin embargo, pese a tener presentes los aspectos pragmáticos en su modelo, habrá que esperar a la segunda revisión del mismo llevada a cabo por Tomás Albaladejo en la «TeSWeST ampliada II» para encontrar un componente explícito de pragmática textual.

\subsubsection{Las macroestructuras y las superestructuras de $\mathbf{T}$. A van Dijk. Macroestructuras pragmáticas y macroactos de habla}

Teun A. van Dijk presenta su teoría de las macroestructuras y superestructuras ya en 1972, en Some Aspects of Text Grammars, y, tras de sarrollarla en diversos trabajos ${ }^{49}$, llegará a ocuparse monográficamente de ella en su

4) T. A van Dijk, Some Aspects of Text Grammars, cit., pp. 17,38 y ss., 130-162, 273 y ss.; «Frammaires textuelles et structures narratives», en c. Chabrol (ed.), Semiotique narrative ef textuelle, Paris, Larousse, 1973, pp. 177-207; (1972), Per una poetica generativa, Bologna, II Mulino, 1976, pp. 66-69; (1973), «Nota sulle macrostructture linguistiche», en M.E. Conte (ed.), La linguistica testuale, cit., pp. 181-194; «Aspectos de una teoría generativa del texto poético», en A. J. Greimas (ed.), Ensayos de semiótica poética, Barcelona, Planeta, 1976, pp. 239-271; «Narrative Macrostructures and Knowledge Frames in Discourse Comprehension», en M. Just y P. Carpenter (eds.), Cognitive Processes in Comprehension, Hillsdale, N. J., Erlbaum, 1977; (1977), Texto y contexto, Madrid, Cátedra, 1980, pp. 195-238; (1980), Estructuras y funciones del discurso, Madrid, Siglo XXI, 1983; «Recalling and Summarizing Complex Discourse», en W. Burghardt - K. Hölzer (comps.), Text Processing, Nueva York/Berlin, de Gruyter, 1978, pp. 49-118; La ciencia del texto: un enfoque interdisciplinario, cit., pp. 54-78, 141-174; T. A. van Dijk y W. Kintsch, «Cognitive Psycology and Discourse: Recalling and Summarizing Stories», en W. U. Dressler (ed.), Current Trends in Textlinguistics, cit., pp. 61-80; «La pragmática de la comunicación literaria», cit. 
Macrostructures $^{50}$. En la hipótesis de macroestructura de van Dijk se halla el punto más importante de sus propuestas de gramática textual.

Van Dijk sitúa las macroestructuras en el nivel semántico, siendo la macroestructura semántica la «estructura profunda textual», esto es, «una representación abstracta de la estructura global de significado de un texto ${ }^{51}$. El concepto de macroestructura es la reconstrucción teórica de nociones intuitivas como la de «tema», «tópico», «esencia» o «asunto» y hace referencia, por tanto, al contenido global de un discurso - o de un fragmento considerado como un todo- y no al de las oraciones individuales, ámbito éste (el de las estructuras reales de las oraciones y de las interrelaciones entre ellas) que corresponde a la microestructura o estructura de superficie textual. Las macroestructuras resultan ser secuencias de (macro)proposiciones, ordenadas y coherentes linealmente y, globalmente, con la macroproposición de base del texto o tópico textual ${ }^{52}$, del que se derivan todas las demás.

Un paso más allá del establecimiento de estructuras semánticas globales, es posible organizar estas macroestructuras asignando funciones a las proposiciones de una secuencia, es decir, asignando proposiciones a categorias ${ }^{53}$ de naturaleza funcional que definen la clase de relación que mantienen con otras proposiciones. Algunas de esas funciones se han convencionalizado en una cultura determinada, dando lugar al establecimiento de esquemas fijos para el

51) T. A. van Dijk, Macrostructures. (An interdisciplinary Study of Global Structures), Hillsdale, N. J., Eribaum, 1980. Con relación a las macroestructuras, vid. también A. García Berrio - T. Albaladejo, «Estructura composicional. Macroestructuras», en Estudios de Lingüística, I, 1983, pp. 127-180; T. T. Ballmer, «Macrostructures», en T. A. van Dijk (ed.), Pragmatics of Language and Literature, 2, Amsterdam, North-Holland Publishing, Company, 1976, pp. 1-22.

5t T. A. van Dijk, La ciencia del texto, cit., p. 55.

52 Sobre el concepto de tópico del texto o tópico del discurso, fundamental en la teoría de las macroestructuras y, en general, en la lingüístico-textual, además de T. A. van Dijk, Texto y contexto, cit., pp. 197 y ss.; La ciencia del texto, cit., pp. 58-59; Estructuras y funciones del discurso, cit., pp. 43-47, vid. E. Agricola, «Vom Text zum Thema», en F. Dane_-D. Viehweger (eds.), Probleme der Textgrammatik, Berlin, Akademie Verlag, 1976, pp. 14 y ss.; Z. Bánréti, «The Topic of Texts and the Interpretation of Texts», en J. S. Petöfi (ed.), Text vs. Sentence Continued. Papiere zur Textlinguistik, 29, Hamburg, Buske, 1982, pp. 43-57; E. Bernárdez, Introducción a la lingüística del texto, cit., pp. 151 y ss.; T. Albaladejo, «Estructura de sentido, representación textual semánticointensional y tópico textual», en Anales de la Universidad de Murcia. Letras 53, 1-2, pp. 268 y ss.; A. Gacía Berrio - T. Albaladejo, «Estructura composicional. Macroestructuras», cit., pp. 145 y ss.; F. Chico Rico, Pragnática y construcción literaria, cit., pp. 74-92; «La organización del material semántico-intensional en la macroestructura del texto literario», en Folia Linguistica, XXV/1-2, 1990, pp. 147-148.

53 Van Dijk utiliza las categorías narrativas de W. Labov y J. Waletsky, «Narrative Analysis. Oral versions of personal experience», en June Helm (ed.), Essays on the Verbal and Visual Arts, Seattle, Washington U. P., 1967, pp. 12-44, apud T. A. van Dijk, Estructuras y funciones del discurso, cit., p. 53 . 
contenido global de un texto ${ }^{54}$. Para diferenciarlas de las macroestructuras, van Dijk llama a este tipo especial de estructuras (no específicamente lingüísticas, aunque se proyectan en estructuras semánticas) que operan también globalmente y están conectadas con aquéllas (siendo distintas de ellas), «superestructuras (esquemáticas)» -también «esquemas convencionales» (conventional schemata) o simplemente «esquemas» (schemata)-. Mientras que la macroestructura es la representación conceptual que del asunto o tema principal del texto tiene el productor, la superestructura es la representación conceptual del tipo de texto. Una superestructura es «un tipo de forma del texto, cuyo objeto, el tema, es decir: la macroestructura, es el contenido del texto» ${ }^{55}$.

Para las categorías superestructurales, van Dijk considera posible hablar no sólo de funciones textuales, las cuales definen relaciones entre proposiciones de un texto (tales como 'preparación', 'explicación', 'especificación', 'contraste', 'comparación' o 'ejemplo', que caracterizan también el nivel microestructural textual), o, en niveles globales, entre secuencias de proposiciones, sino también de funciones pragmáticas, cognitivas y socioculturales (pensemos, por ejemplo, en el papel que juegan las argumentaciones en procesos de persuasión). La estrecha vinculación que van Dijk sugiere entre los esquemas superestructurales y los esquemas pragmáticos e interaccionales puede dar lugar en secuencias a interacciones estereotipadas (así, por ejemplo, el desarrollo de una asamblea o mitin).

Pero tanto las macroestructuras semánticas como las superestructuras dependen en última instancia del contexto pragmático ${ }^{56}$ : las emisiones se «usan» en contextos de comunicación e interación sociales y tienen, por consiguiente, funciones específicas en tales contextos como «acciones», actos de habla. T. A. van Dijk define las macroestructuras pragmáticas como aquellas estructuras globales del discurso que se explican en términos de acción, de interacción, y, en particular, de actos de habla y del contexto pragmático en el cual son apropiados o no. Se trata de estructuras de actos globales que se asignan a una secuencia de actos de habla que van Dijk llama macroactos de habla. Un macroacto de habla es un acto de habla que resulta de la realización de una secuencia de actos de habla conectados.

De la misma manera que el nivel pragmático engloba al semántico, las macroestructuras pragmáticas englobarían o contendrían a las semánticas: un

54 Van Dijk se refiere a la naturaleza de los factores que subyacen a la formación y convencionalización (incluso institucionalización) de esquemas en T. A. van Dijk, Macrostructures, cit., p. 107-108.

5.5 T. A. van Dijk, La ciencia del texto, cit., p. 142.

56. Afirma van Dijk: «[...] all information in a text and, hence, the macrostructures should be interpreted relative to other information in the text and relative to information of the context, both in the perspective of interdependency and in that of functionality» ( T. A. van Dijk, Macrostructures, cit., p. 128). 
acto de habla global debe, después de todo, tener también un «contenido global», un tema o tópico. Los macroactos de habla proporcionan, en consecuencia, la necesaria contrapartida pragmática de las macroestructuras semánticas. De la misma manera que hablamos de «tema» o «tópico» de un discurso, gracias al concepto de 'macroestructura pragmática' se puede hacer explícito lo que podríamos llamar el «propósito pragmático» del mismo. Ahí radica la importancia de las nociones de 'macroacto de habla' y 'macroestructura pragmática' para una gramática del texto y para una teoría más general del discurso: en que hacen posible hablar de las «funciones globales» de un discurso o de una conversación ${ }^{57}$.

La propuesta inicial de Teun A. van Dijk de una gramática textual semánticamente fundamentada en el concepto de 'macroestructura' experimenta una ampliación progresiva de sus límites al conceder van Dijk cada vez mayor importancia a los aspectos psicológico-cognitivos y sociales del lenguaje y la comunicación. La ampliación del concepto de 'macroestructura' del ámbito semántico al pragmático supone el paso de una gramática textual a una Teoría del texto pragmáticamente fundamentada, paralelamente a como la evolución de la linguiística textual (o de la Lingúística a través de la lingüística textual) supone la apertura de «lo tradicional lingüístico» a los factores psicológicos y sociales, pragmáticos en general de la comunicación.

\subsubsection{Aspectos pragmáticos de la TeSWeST}

El lingüista húngaro János S. Petöfi anuncia ya en $1971^{58}$ su teoría general del texto, que conocemos con el nombre abreviado de TeSWeST — del alemán Text-Struktur Welt-Ŝtruktur Theorie- o teoría de la estructura del texto y de la estructura del mundo, de la cual presenta un bosquejo en Neuchâtel en 1973 y cuyo desarrollo podemos seguir en diversos trabajos ${ }^{5 \%}$. Cuestiones que habían

57 Cr. T. A. van Dijk, Some Aspects, cit., pp. 313-342; Texto y contexto. Semántica y pragmática del discurso, Madrid, Cátedra, 1980, pp. 290-324; Macrostructures, cit., pp. 175-199; Studies in the Pragmatics of Discourse. The Hague, Mouton, 1981; Estructuras y funciones del discurso, cit., pp. 58-76, 77-114; La ciencia del texto, cit., pp. 79-107.

58 J. S. Petöft, Transformationsgrammatiken und eine ko-textuelle Texttheorie, Frankfurt, Athenäum, 1971; «'Generativity' and Text-Grammar», en Folia Linguistica, V, 1971, pp. 277-309.

5) Vid. J. S. Petöfi, «Towards an Empirically Motivated Grammatical Theory of Verbal Texts», en J. S. Petöfi - H. Rieser (eds.), Studies of Text Grammar, Dordrecht, Reidel, 1973, pp. 205-275; Vers une thếrie partielle du texte, Hamburgo, Helmut Buske, 1975; «Semantica, pragmatica, teoria del testo», en M. E. Conte (ed.), La linguistica testuale, cit., pp. 195-223; «Osservazioni sul componente grammaticale d'una teoria semiotica integrata dei testi», ibid., pp. 224-247; «La teoría lógico-semántica de las lenguas naturales como teoría textual (Programa de investigación para lingüística formal y lógica natural)», en J. S. Petöfi - A. García Berrio, Lingüística del Texto y Crítica Literaria, Madrid, Comunicación, 1978, pp. 99-125; «Una teoría textual formal y semiótica como teoría integrada del lenguaje natural. (Notas metodológicas)», ibid., pp. 127-145; «Estructura y función del componente gramatical de la teoría de la estructuta del texto y de la estructura del mundo», ibid., pp. 147-189; «Léxico, conocimiento enciclopédico, teoría del texto», ibid., pp. 191 - 
sido sólo apuntadas o contempladas de manera intuitiva en investigaciones linguístico-textuales precedentes alcanzan finalmente forma teórica en el modelo de Petöfi.

La TeSWeST, en tanto teoría semiótica que es, ha de cubrir, además de los aspectos sintácticos y semánticos - que hemos visto hasta ahora-, también los aspectos pragmáticos de los textos de lengua natural. En $1979^{60}$ Petöfi revisa de nuevo su teoría e introduce un modelo de comunicación de gran utilidad para el tratamiento de la Pragmática dentro de la TeSWeST, manteniendo sin embargo el esquema básico del modelo: el formado por los componentes gramatical, semántico-extensional y léxico. Los aspectos pragmáticos son tratados, pues, implícitamente dentro de estos tres componentes fundamentales, pues, pese a ser la TeSWeST una teoría (del texto) formal semánticamente/pragmáticamente basada, Petöfi no cree posible la creación en el seno de su teoría de un componente pragmático independiente:

no independent pragmatic component can be separated within a theory of language. This means that the pragmatic parameters must be treated together with the others parameters to be reconstructed by this theory of language ${ }^{61}$.

La TeSWeST permite dar cuenta, por tanto, de los aspectos cotextuales (los que atañen a la estructura gramatical: fono-fonológicos, morfosintácticos y semántico-intensionales), así como de los contextuales (los concernientes a la semántica extensional y los aspectos pragmáticos) ${ }^{62}$, y lo hace no sólo en la dirección de producción o síntesis (WeS - TeS) sino también en la de recepción o análisis (TeS -WeS). Esta bidireccionalidad analítico-sintética que caracteriza la organización de los componentes de la TeSWeST es en sí un hecho que está apuntando el carácter pragmático del modelo. Lo mismo sucede con la importancia concedida al contexto dentro de la teoría: uno de los dos grandes

213; «La representación del texto y el léxico como red semántica», ibid, pp. 215-242. Para una presentación o síntesis del modelo, vid. A. García Berrio, «Situación de la teoría textual (La teoría de J. S. Petöfi en el marco de la lingüística del texto), ibid., pp. 55-98; A. García Berrio - A. Vera Luján, Fundamentos de Teoria Lingüística, cit., pp. 191-223; T. Albaladejo - A. García Berrio, «La Lingüística del Texto", en F. Abad - A. García Berrio (eds.), Introducción a la lingüústica, Madrid, Alhambra, 1982, pp. 233-247.

6n J. S. Petöfi, «La estructura de la TeSWeST. Aspectos de la interpretación pragmáticosemántica de textos de lengua objeto», contribución al II Curso de Lingüística Textual, Universidad de Murcia, 21-28 de marzo de 1979, mecanografiado; «Einige Grundfragen der pragmatischsemantischen Interpretation von Texten», en T. Ballmer - W. Kindt (eds.), Zum Thema 'Sprache und Logik': Ergebnisse einer interdisziplinären Diskussion, Hamburg, Buske, 1980.

61 J. S. Petöfi, «Formal Pragmatics and a Partial Theory of Texts, en S. J. Schmidt (Hrsg.), Pragmatik/2, München, Fink, 1976, p. 111.

62 Mientras que la semántica intensional tiene como objeto de estudio «las relaciones semánticas intersígnicas e intrasígnicas» (T. Albaladejo, Teoría de los mundos posibles y macroestructura narrativa. Análisis de las novelas cortas de Clarín, Alicante, Universidad de Alicante, 1986, p. 18), la semántica extensional o referencial (también llamada semántica del mundo) «tiene su área de estudio en las relaciones entre el referente y el signo lingüístico que lo expresa» (Ibid., p. 18). 
bloques del modelo es propiamente contextual (el de la «la teoría del mundo», WeS); además, las cuestiones contextuales llegan a introducirse en las cotextuales o gramaticales, siendo objeto de consideración lingüística lo propiamente extralingüístico en la medida en que es textualizado. De esta forma, los aspectos pragmáticos están presentes en todos los componentes del modelo, incluso en el menos pragmático por definición.

Explícitamente, Petöfi sólo se ocupa de cuestiones pragmáticas parciales. Para él es el léxico el componente de la TeSWeST con mayor relieve pragmático. Puesto que las representaciones explícitas de las intensiones (los definientes) describen el modo en que los miembros de una comunidad lingüística utilizan las palabras de la lengua en cuestión, «La TeSWeST offre perciò (fra altro) la descrizione sincronica della conoscenza pragmatica correlata allo stock lessicale di una data lingua o sottolingua) naturale ${ }^{63}$.

Respecto al tratamiento de aspectos pragmáticos en el componente de gramática del texto, basándose en la consideración por parte de ciertas concepciones teóricas de la Pragmática como «pragmática formal» (de naturaleza cotextual), alude Petöfi a las descripciones, cotextuales, de índices o elementos deícticos, donde tienen cabida todas las consideraciones sobre su uso anafórico y catafórico.

Mayor es la relevancia pragmática del componente de semántica del mundo (por su carácter contextual, ya señalado), destacando Petöfi al respecto los siguientes rasgos: (a) los índices contextuales (que señalan la persona, el lugar o el tiempo del referente); (b) los parámetros, en las definiciones del léxico, que dependen del contexto (expectativas, sistema de normas, etc.); (c) la elipsis contextual; (d) las intensiones específicas del contexto no derivables de las intensiones fijadas en el léxico (incluye el uso en sentido figurado de las expresiones verbales).

A partir de la TeSWeST de János S. Petöfi, Tomás Albaladejo elabora la «TeSWeST ampliada I» y la «TeSWeST ampliada II ${ }^{64}$, dos nuevas versiones que suponen el enriquecimiento del modelo inicial de Petöfi. Es en la segunda de ellas, la versión «ampliada II», en la que Tomás Albaladejo se ocupa específicamente del aspecto pragmático del modelo.

La «TeSWeST ampliada II» incorpora al modelo lingüístico-textual un componente explícito de pragmática textual. Aunque los aspectos pragmáticos están

Respecto a los conceptos de intensión y extensión, vid.: R. Carnap, «Significación y sinonimia en las lenguas naturales», en E. Coumet - O. Ducrot - J. Gattegno (eds.), Lógica y lingüística, Buenos Aires, Nueva Visión, 1978, pp. 111-125; B. Stanosz, «Formal Theories of Extension and Intension of Expression», en Semiotica 2, 1970, pp. 102-114. Sobre la sintaxis, la semántica y la pragmática como integrantes de la semiótica, vid. Ch. W. Morris, «Fundamentos de la teoría de los signos», cit.

63 J. S. Petöfi, «Semantica, pragmatica, teoria del testo», cit., p. 219.

64 Respectivamente: T. Albaladejo, «Aspectos del análisis formal de textos», Revista Española de Lingüística, XI, 1, enero-junio 1981, pp. 117-160; T. Albaladejo, «Componente pragmático, componente de representación y modelo lingüístico-textual», Lingua e Stile, XVII, 1, gennaiomarzo 1983, pp. 3-46. 
presentes en la TeSWeST estándar y en la primera versión ampliada de Albaladejo (no olvidemos que Petöfi concibe su teoría de la estructura del texto y de la estructura del mundo como teoría semiótica), éstos son tratados dentro de los componentes fundamentales del modelo, pues no hay, como hemos visto ya, un componente específicamente pragmático. Dado que la Pragmática es la base de la Semántica y la Sintaxis, considera Albaladejo que es conveniente la elaboración de un componente pragmático que, englobando los demás componentes del modelo, constituya junto a ellos un modelo de comunicación lingüística a la vez que recoja de modo explícito todos los aspectos pragmáticos del lenguaje. De esta manera, la base pragmática del modelo semiótico de la TeSWeST quedará explicitada mediante el componente de primer grado de pragmática textual (CIPT) de la TeSWeST ampliada II, que gobierna todos y cada uno de los procesos de los que da cuenta el conjunto jerarquizado de los componentes que integran el modelo lingüístico-textual. En palabras de Albaladejo, el nuevo C1PT «[...] permite y explica la realización del conjunto de procesos categoriales mediante los que se produce la comunicación lingüística y, consiguientemente, la categoría acto de comunicación lingüística [participación de un mensaje, texto de lengua natural, por el productor normal al receptor normal en un contexto, a través de un canal y mediante un código lingüístico común a los comunicantes], y explica la realización de los procesos particulares de comunicación y, por tanto, los actos de comunicación lingüística concretos. $\rangle^{65}$

En la TeSWeST ampliada II están reflejadas, por un lado, la relación de la Pragmática con la Sintaxis y la Semántica; por otro, la relación entre Pragmática lingüística y lingüística textual. Respecto a la primera, el C1PT integra, a través de los componentes de segundo grado de producción textual - $\mathrm{C} 2 \mathrm{PrT}-\mathrm{y}$ de recepción textual $-\mathrm{C} 2 \mathrm{ReT}-$, los componentes de extensión textual, C3ET, y de intensión textual, C3IT, -y todos sus (sub)componentes y categorías-, de la misma manera que la Pragmática integra la Semántica y a la Sintaxis. Respecto a la segunda, el componente pragmático está integrado directamente en el marco general del modelo lingüístico-textual, puesto que «el 'acto expresivo' que origina como componente pragmático el texto es la energía que produce un resultado lingüístico, el texto que la recubre», de modo que «desde un texto en concreto puede reconstruirse la estructura del acto expresivo en todos sus pasos $»^{66}$. La TeSWeST ampliada II reproduce intuitiva y formalmente la competencia lingüístico-textual comunicativa del productor/receptor común de una lengua natural; es un modelo textual semiótico de base pragmática.

65 T. Albaladejo, «Componente pragmático, componente de representación y modelo lingüístico-textual», cit., p. 23.

${ }_{66}$ A. García Berrio, «Lingüística, literaridad/poeticidad (Gramática, pragmática, texto)», 1916. Anuario de la Sociedad Española de Literatura General y Comparada, II, 1979 (1980), p. 146. 
La TeSWeST (estándar) de János S. Petöfi y especialmente la TeSWeST ampliada II de Tomás Albaladejo representan el estadio más avanzado en el desarrollo de la lingüística textual como tal, en tanto que disciplina «total»e integradora que pretende dar cuenta de la estructura y el funcionamiento del texto (unidad básica de intercambio comunicativo) en la comunicación y de la comunicación en el texto.

Como teoría textual, la TeSWeST ampliada II es un reflejo de la competencia textual-comunicativa de los hablantes. Como teoría semiótica pragmáticamente fundamentada, pretende explicar todos los aspectos (sintácticos, semánticos y pragmáticos, como fundamento de los dos anteriores) de los textos de lengua natural y explicar el proceso comunicativo, desde la perspectiva del productor al igual que desde la del receptor del mismo, en, por y desde el texto.

La TeSWeST ampliada II es una manifestación clara de la ampliación que experimenta el campo de la Lingüística con la consecución del nivel textual: la realidad externa al texto (social, psicológica, cultural) se convierte en objeto de estudio de la Ciencia lingüística en la medida en que ésta es textualizada/ textualizable o determina las características del texto.

\section{Conclusiones}

Tras los primeros intentos de acercamiento al 'texto' — todavía desde la oración-, amparados sobre todo por el estructuralismo, y las primeras tentativas de gramáticas textuales y de definición de la unidad textual como unidad semántica global en el marco teórico ofrecido por el generativismo, el proceso de búsqueda de la identidad textual y de fundamentación de la Lingüística en el texto culmina con la apertura de la Lingüística a la Pragmática y, con ella, a los aspectos contextuales y comunicativos del lenguaje. La evolución de la lingüística textual -y propuestas teóricas como la de van Dijk o la de Petöfi son un claro ejemplo de ello- demuestra que la Gramática no puede ser vista independientemente de la Pragmática, sino que, al contrario, su papel en el estudio del texto o discurso será tanto más adecuado y relevante cuanto más esté fundamentada sobre presupuestos pragmáticos. Los modelos lingüísticotextuales sucintamente expuestos, ponen de manifiesto el interés, natural dado su preocupación por el lenguaje-, de la Lingüística (textual) por lo que la Pragmática significa: el «lenguaje en uso», la vertiente comunicativa del lenguaje, a través del texto, en tanto que unidad lingüística - y comunicativa-básica; y especialmente las teorías de van Dijk y Petöfi representan el estadio más avanzado en la evolución de la lingüística textual, su consolidación gracias a la Pragmática y gracias a la consecución, por el papel mediador de la Pragmática -integrador, en este caso, de la Sintaxis y la Semántica, completando así la tríada morrisiana-, de la dimensión semiótica del texto, en el texto. 
Al trasladar el interés de la estructura del texto, es decir, de la unidad textual en sí misma, a la dimensión comunicativa del texto, la lingüística textual entra a considerar el contex to del texto, o el tex to en su contexto, oscilando entre dos polos fundamentales: la función social de la lengua (la cual es resultado de la interacción de individuos que están inmersos en un marco colectivo, social) y los procesos psicológicos del individuo (por los que se había interesado la lingüística mentalista de Noam Chomsky, en tanto que todos los individuos pueden realizar un conjunto de procesos psíquicos similares). La Lingüística del texto representa la síntesis de lo propiamente textual y lo pragmático o contextual, de lo «lingüístico» y lo «extralingüístico» en el 'texto', unidad fundamental de la comunicación humana.

La asunción previa de la textualidad facilitó, sin duda, el acceso de y a la Pragmática. Sirva como ejemplo el ámbito de los estudios literarios, en los cuales la perspectiva pragmática pronto echó raíces y desde entonces no ha dejado de cosechar frutos, desde ópticas diversas. Tanto la lingüística textual como la Ciencia literaria en general (especialmente las orientaciones teóricoliterarias y/o crítico-literarias que surgen de la colaboración de ambas) han desempeñado, pues, un papel muy importante en el establecimiento de la Pragmática como campo de estudio o, incluso, disciplina.

La expansión o ampliación de los límites de la Lingüística, por una parte, mediante la consecución del nivel textual; por otra, a partir de - o unido aello y por influencia de la Pragmática, mediante la apertura a la consideración de los aspectos contextuales del lenguaje, básicamente sociales y psicológicos, comunicativos en general, hace de la Lingüística ciencia integradora, territorio de confluencias de múltiples disciplinas con el texto como eje: Ciencia del texto $^{67}$.

67 S. J. Schmidt, (1973), Teoría del texto. Problemas de una lingüistica de la comunicación verbal, cit.; T. A. van Dijk, La ciencia del texto: un enfoque interdisciplinario, cit.; R. A. de Beaugrande, «Teoría linguística y metateoría para una ciencia del texto», en E. Bernárdez (comp.), Lingüistica del texto, Madrid, Arco, 1987, pp. 35-94. A la necesidad de una Ciencia del texto puede decirse que responden propuestas como la «Textología semiótica» de J. S. Petöfi (cf. J. S. Petöfi, «Report: European Research in Semiotic Textology. A Historical, Thematic, and Bibliographical Guide», en Folia Linguistica, 20, 1986, pp. 545-571; «Constitution and Meaning: A Semiotic TextTheoretical Approach», en M. E. Conte - J. S. Petöfi - E. Sözer (eds.), Text and Discourse Connectedness, Amsterdam/Philadelphia, John Benjamins Publishing, 1989, pp. 508-542; Towards a Semiotic Theory of the Human Communication (Text Linguistics-Semiotic Textology), Macerata, Universidad, 1991; J. S. Petofi (ed.), Sistemi segnici e loro uso nella comunicazione umana 1: Aspetti generali. Quadro interdisciplinare della ricerca (Quaderni di Ricerca e Didattica, IX), Macerata, Universidad, 1993; J. S. Petofi (ed.), Sistemi segnici e loro uso nella comunicazione umana 2: La filosofia del linguaggio e la comunicazione umana multinediale (Quaderni di Ricerca e Didattica, XIV), Macerata, Universidad, 1995, y, sobre todo, la ciencia general del discurso o «Retórica General» de A. García Berrio (cf. A. García Berrio, «Retórica como ciencia de la expresividad. (Presupuestos para una Retórica General)», en Estudios de Lingüística, 1, 1983, pp. 127-180). 
Así pues, la lingüística textual, desde y en el texto, alcanza e integra el ámbito general de la comunicación, confluyendo con la Pragmática lingüística en lo que Siegfried J. Schmidt denomina «juego de acción comunicativo», que está en la base de un nuevo, complejo concepto de texto, en torno al cual se construye la nueva Teoría o Ciencia del texto.

Ciertamente los saberes que se han dado en llamar pragmáticos poseen una tradición milenaria retórica que entronca con las tradiciones filológicas ligadas a la Historia, la Teoría y la Crítica literarias, y, contemporáneamente, a los estudios socilógicos desde sus versiones marxistas hasta los más actuales y diversos desarrollos. Igualmente milenaria es por la Retórica la tradición de atención al discurso, en la cual hunden sus raíces todas aquellas disciplinas que, por uno u otro camino, hacen del texto su objeto de estudio. Ciertamente es la Retórica la ciencia general, antigua, nueva, eterna del lenguaje y del discurso. 